Proceedings of the $2^{\text {nd }}$ ICEENG Conference, 23-25 Nov. 1999

\begin{tabular}{|l|l|}
\hline $\mathrm{BE}-4$ & 1 \\
\hline
\end{tabular}

Military Technical College
Kobry Elkobbah,
Cairo, Egypt

\title{
Optimum Detection of The Fetus Heart ECG Signal
}

\author{
E.A. Solit(Ph.D.)* M.E.Gadallah(Ph.D.) ${ }^{* *}$ and A.Salah(Eng.) $)^{* * *}$
}

\section{ABSTRACT:}

In the physiological process, the desired signal may not be directly measurable, and the investigator may not be able to determine the signal from measurable composite signals, such as the case of the fetus ECG (F-ECG). There is a problem in extracting the F_ECG signal from the composite maternal ECG signal obtained from the abdominal lead, because the Interference maternal ECG (M-ECG) is stronger than the fetus ECG signal. In this paper, the adaptive noise canceller is presented, to detect both the maternal and fetal ECG signals separately. The proposed scheme exhibits a good performance and the maternal and fetal ECG is successfully detected.

\section{I-INTRODUCTION}

The fetal electrocardiogram (FECG) was first measured in 1906 by Cremer using external abdominal electrodes. Yet the use of FECG in fetal heart rate (FHR) determination, and the subsequent clinical utilization of the fetal heart rate in monitoring fetal development and responsiveness, have occurred only relatively recently [1]. Abdominal electrocardiograms make it possible to determine the fetal heart rate and to detect multiple fetuses, are often used during labor. The background noise due to muscle activity and fetal motion, however, often has amplitude equal to or greater than that of the fetal heart beat. A still more serious problem is the mother's heartbeat, which has amplitude 2 to 10 times greater than of the fetal heartbeat and often interferes with its recording. Four ordinary chest leads were used to record the mother's heartbeat and provide multiple reference input to the canceller. A single abdominal lead was used to record the combined maternal and fetal heartbeats that served as primary input [2]. Measurements will performed on a pregnant women who has a gestation period of 20-37 weeks. The largest FECG amplitude are recorded between 20 and 28 weeks and after 32 weeks of gestation.[3] In this paper a proposed scheme for detecting the fetus ECG signal from the composite maternal ECG signal is introduced. This algorithm depends on using the adaptive noise canceller. The paper is organized as follows. The system configuration in section II, a proposal scheme of an adaptive ECG detector in section III, the simulation results are described in section IV, conclusion are described in section $\mathrm{V}$.

${ }^{*}$ Ass. prof.,Military Technical colleage

${ }^{\star \star}$ Ass. prof., Military Technical colleage

${ }^{\star \star \star \star E n g . ~ M . S c ., ~ M i l i t a r y ~ T e c h n i c a l ~ c o l l e a g e ~}$ 


\section{II-Adaptive Noise Canceling}

The subject of adaptive noise canceling is introduced and is treated extensively by Widrow et al. [4]. Fig. 1 shows a block diagram of an adaptive noise canceling system There are two inputs; the primary input contains signal plus additive rioise, and the reference input contains noise alone.

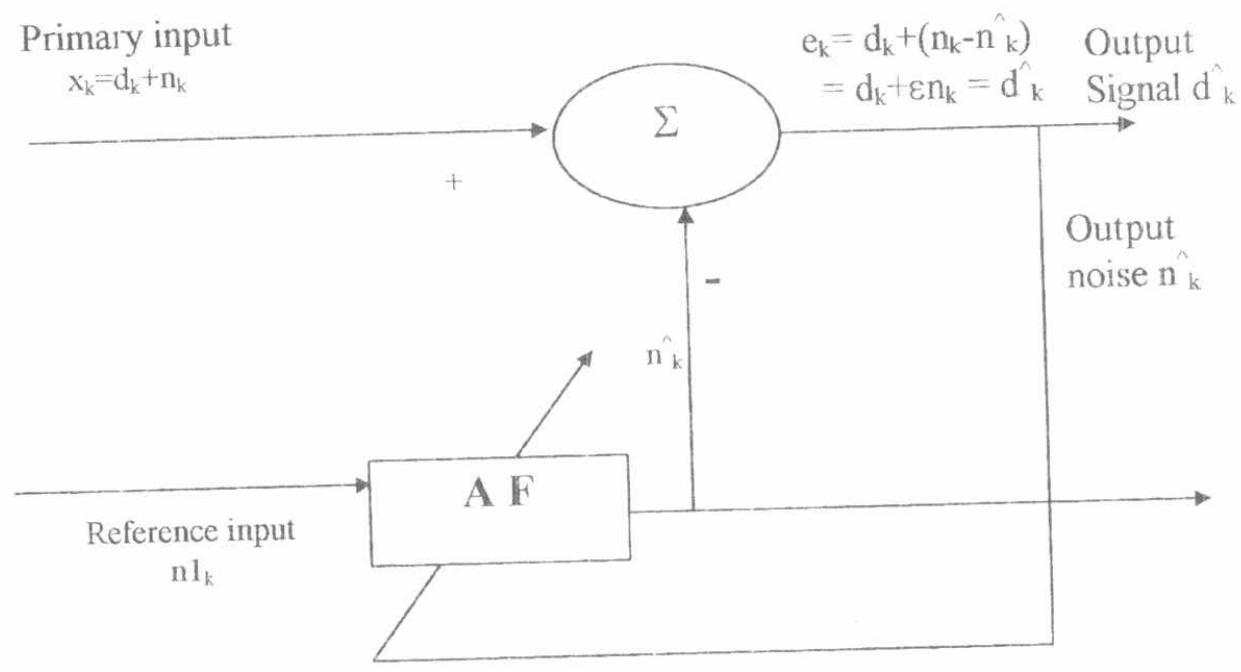

Figure 1 block diagram of an adaptive noise canceller

\section{Purpose of the adaptive filter}

The purpose of the adaptive filter is to estimate the additional noise signal $n_{k}$ and generate a good replica of the assigned noise signal $n_{k}$. The filter coefficient is updated according to the Least Mean Square (LMS) adaptation algorithm such that the (Mean Square Error) MSE of the residual error signal $\varepsilon n_{k}$ is minimized. The filter output is calculated as follows:

$\hat{\mathrm{n}}_{\mathrm{k}}=\sum_{i=0}^{L} W i * n 1(k-i)$

The error signal can be expressed as:

$e_{k}=x_{k}-\hat{n}_{k}=d_{k}+\left(n_{k}-\hat{n}_{k}\right)=d_{k}+\varepsilon n_{k}$

$\varepsilon n_{k}=n_{k}-W_{k}^{-} N_{1 k}$

Where $W_{k}$ and $N_{1 k}$ are defined as the coefficient and observation vectors respectively.

The mean square error can be written as:

$E\left(\varepsilon_{n k}^{2}\right)=E\left(n_{k}-\left(w_{k}^{\top} N_{1 k}\right)\right)^{2}$ 
$=E\left(n_{k}^{2}\right)-2 E\left(n_{k}-w_{k}^{\top} N_{1 k}\right)+w_{k}^{\top} E\left[N_{1 k} N_{1 k}^{\top}\right] w_{k}$

$=E\left(n_{k}^{2}\right)-2 w_{k}^{\top} P_{k}+w_{k}^{\top} R_{N} w_{k}$

$\mathrm{w}^{*}=\mathrm{R}^{-1}{ }_{\mathrm{N}} \mathrm{P}_{\mathrm{k}}($ Optimal solution of Wiener Hopf equation)

The output signal, $e_{k}$ is defined as:

$e_{k}=x_{k}-\hat{n}_{k}$

\section{III-LMS ADAPTATION ALGORITHM}

The LMS adaptation algorithm can be written as:

$$
\mathbf{W}(\mathbf{k}+1)=\mathbf{W}(\mathbf{k})+2 \mu e_{k} N 1_{k}
$$

Where the parameters are: -

$\begin{array}{llll}W(k+1) & \text { the updating coefficient. } & W(k) & \text { the old coefficient. } \\ \mathrm{u} & \text { the step size. } & e_{k} & \text { the error signal. } \\ N 1_{k} & \text { the observation vector } & & \end{array}$

The error can not be measured directly, so the error output signal $e_{k}$ is used rather than the error signal $\varepsilon n_{k}$, hence the LMS adaptation algorithm can be written as:

$W(k+1)=W(k)+2 \mu \varepsilon_{k} N 1_{k}$

However the output error signal $e_{k}$ includes the useful signal $d_{k}$ and that represents an interference signal to the LMS adaptation algorithm, therefore the adaptive noise canceller works well if and only if the input signal to noise ratio(input SNR) is smaller than $0 \mathrm{~dB}$. i.e. the noise signal is dominant. As the input SNR increases above $0 \mathrm{~dB}$, the performance of the adaptive noise canceller (output SNR) deteriorates.[3]

\section{IV-PROPOSED SCHEME OF AN ADAPTIVE FETUS ECG DETECTOR}

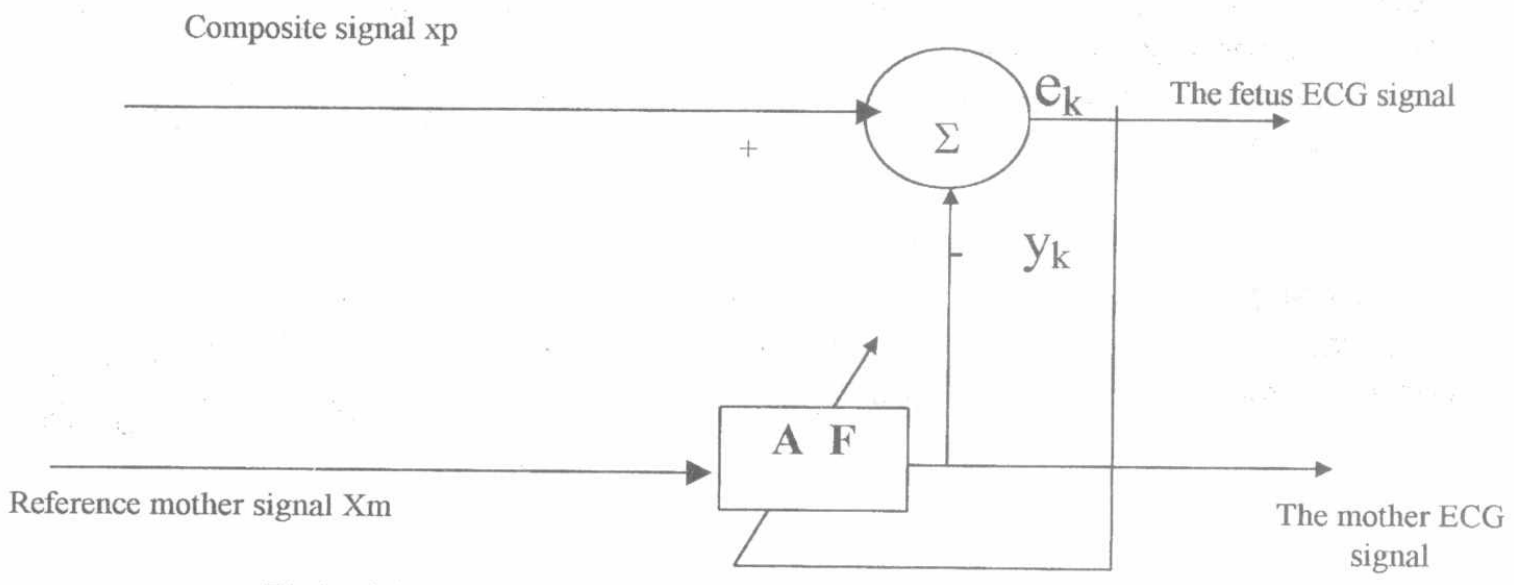

Fig.2. Adaptive fetus ECG detector configuration 
Proceedings of the $2^{\text {nd }}$ ICEENG Conference, 23-25 Nov. 1999

$X m=$ The mother signal:

A $F=$ The Adaptive filter:

$\mathrm{Xp}=$ The composite maternal ECG signal

$y=$ The output mother signal:

ek $=$ The output fetus signal :

The sampling rate chosen for this implementation was about $500 \mathrm{~Hz}$, The adaptation speed $\mu$ were generally chosen to be between 0.02 and 0.2 and the number of coefficient of the adaptive filter $n$ equal 5

\section{V-SIMULATION AND RESULTS:}

In this paper. The mother ECG signal is represented by a real ECG heart signal as depicted in figure (3-a). The composite maternal ECG signal represent the primary signal to the noise canceller as depicted in figure (3-b). The adaptive filter has tow output, the first one is a good estimate of the mother ECG signal as depicted in figure(3-c)and the second one is a good estimate of the fetus ECG signal. The output signal of the adaptive filter in the steady state is considered in the three cases $A, B, \& C$.

Case $A$ : The amplitude of the mother ECG signal is equal to 2 times the fetus ECG signal. The signal to interference ratio(SIR) is equal to $-3 \mathrm{~dB}$, figure 3 illustrates the detected output of the mother ECG signal. Also the output signal $e_{k}$ represents a good estimate of the fetus signal at an output SIR $=5 \mathrm{~dB}$, and the improvement factor is equal to $8 \mathrm{~dB}$.

Case B: The amplitude of the mother ECG signal is equal to 5 times the fetus ECG signal. The signal to interference ratio(SIR) is equal to $-11 \mathrm{~dB}$, figure 4 illustrates the detected output of the mother ECG signal. Also the output signal $e_{k}$ represents a good estimate of the fetus signal at an output SIR $=0 \mathrm{~dB}$, and the improvement factor is equal to $11 \mathrm{~dB}$.

Case C: The amplitude of the mother ECG signal is equal to 10 times the fetus ECG signal. The signal to interference ratio(SIR) is equal to $-16 \mathrm{~dB}$, figure 5 illustrates the detected output of the mother ECG signal. Also the output signal $e_{k}$ represents a good estimate of the fetus signal at an output $S I R=4 d B$, and the improvement factor equal $20 \mathrm{~dB}$.

It's apparent that the proposed scheme of the fetus ECG detector exhibits a good performance and provides large improvement factor in the output signal to noise ratio for both fetus and the mother ECG signals. Hence, The proposed scheme verifies a signal enhancement for both the mother and the fetus ECG signals. 

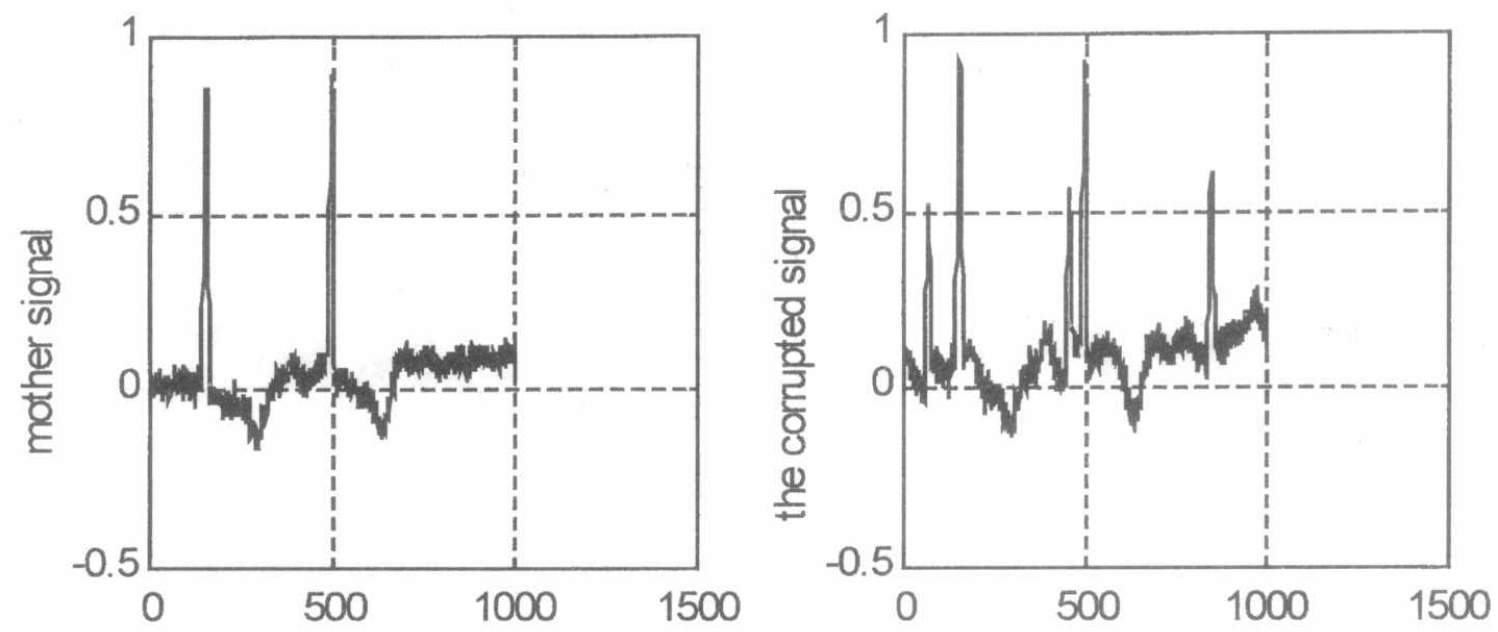

figure a the $i / p$ mother signal time

figure $b$ the comupted fetus signal time
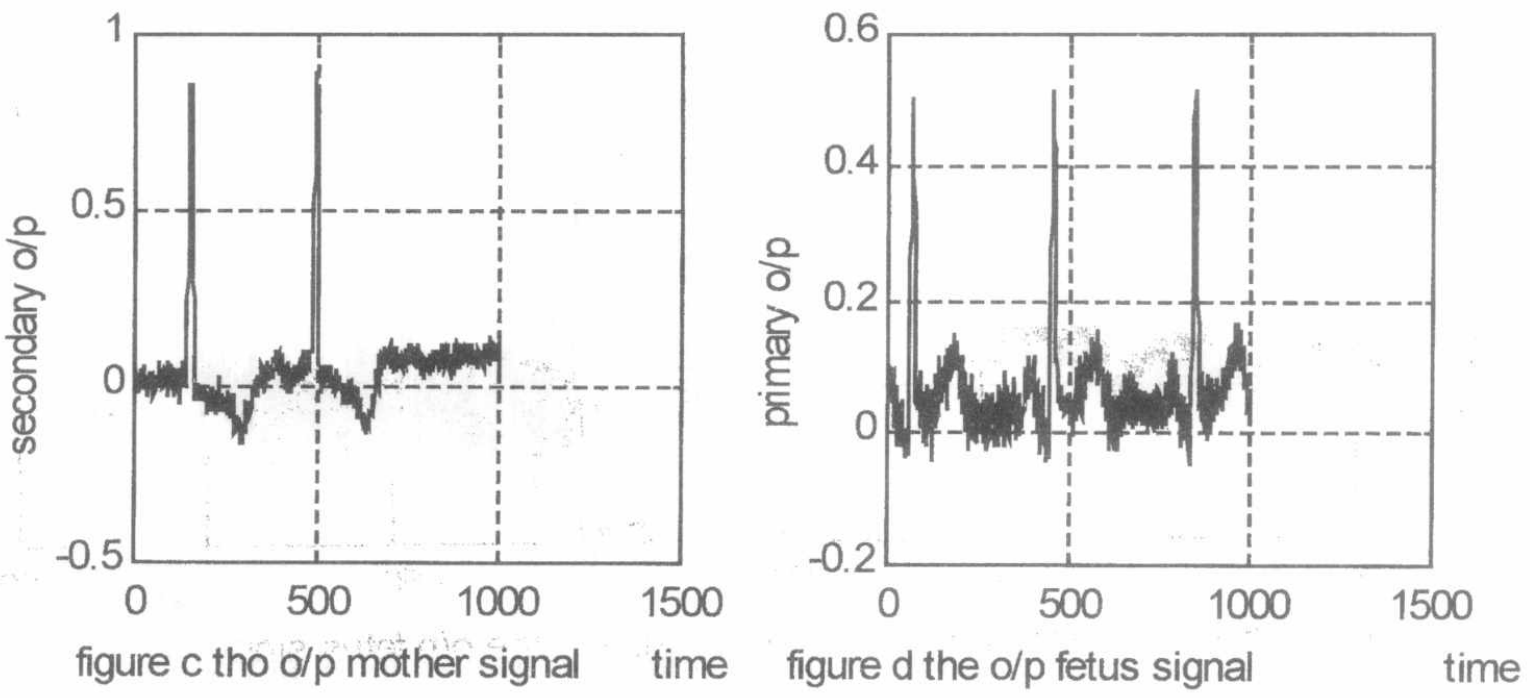

Figure3 The ECG signals waveform for the fetus ECG detector

The mother ECG signal is represented by a real ECG heart signal as depicted in figure (3-a). The composite maternal ECG signal represent the primary signal to the noise canceller as depicted in figure (3-b). The adaptive filter has tow output, the first one is a good estimate of the mother ECG signal as depicted in figure (3-c). And the second one is a good estimate of the fetus ECG signal as depicted in figure (3-d) The mother signal amplitude equal to 2 times than the fetus signal, INSR $=-5 \mathrm{~dB}, \mathrm{OSNR}=3 \mathrm{db}$ and the improvement factor equal $8 \mathrm{~dB}$. 

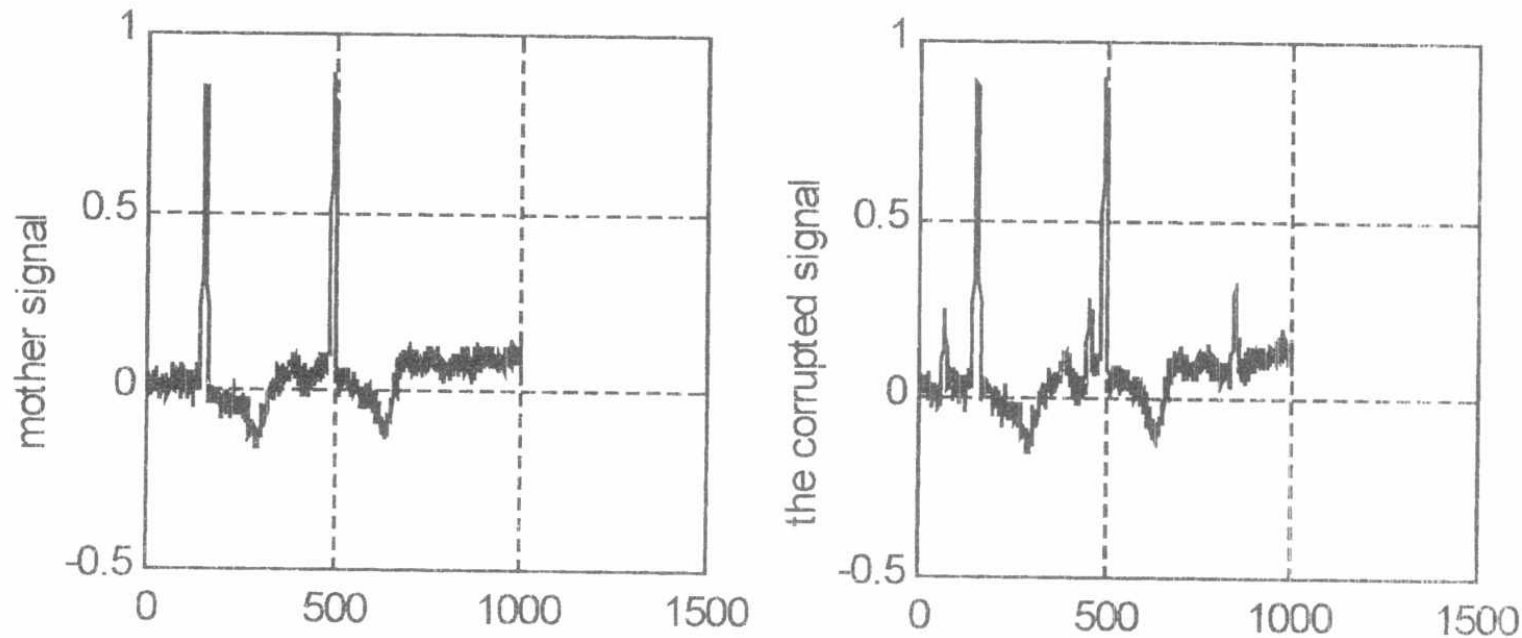

figure a the i/p mother signal time

figure $b$ the comupted fetus signal time
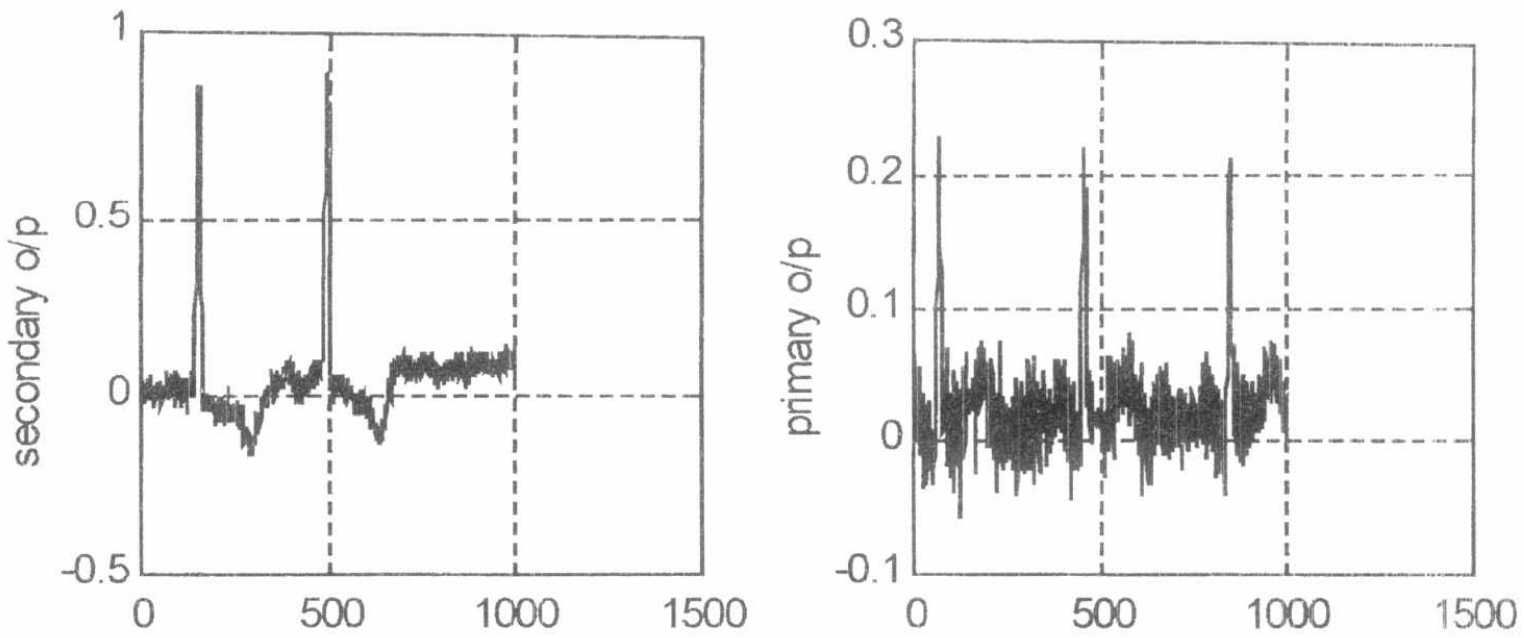

figure $\mathrm{c}$ tho $\mathrm{o} / \mathrm{p}$ mother signal time

figure $d$ the $d / p$ fetus signal

time

Figure 4 The ECG signals waveform for the fetus ECG detector

The mother ECG signal is represented by a real ECG heart signal as depicted in figure (4-a). The composite maternal ECG signal represent the primary signal to the noise canceller as depicted in figure (4-b). The adaptive filter has tow output, the first one is a good estimate of the mother ECG signal as depicted in figure (4-c). And the second one is a good estimate of the fetus ECG signal as depicted in figure (4-d) The mother signal amplitude equal to 5 times than the fetus signal, INSR $=-11 \mathrm{~dB}, \mathrm{OSNR}=0 \mathrm{~dB}$ and the improvement factor equal $11 \mathrm{~dB}$. 

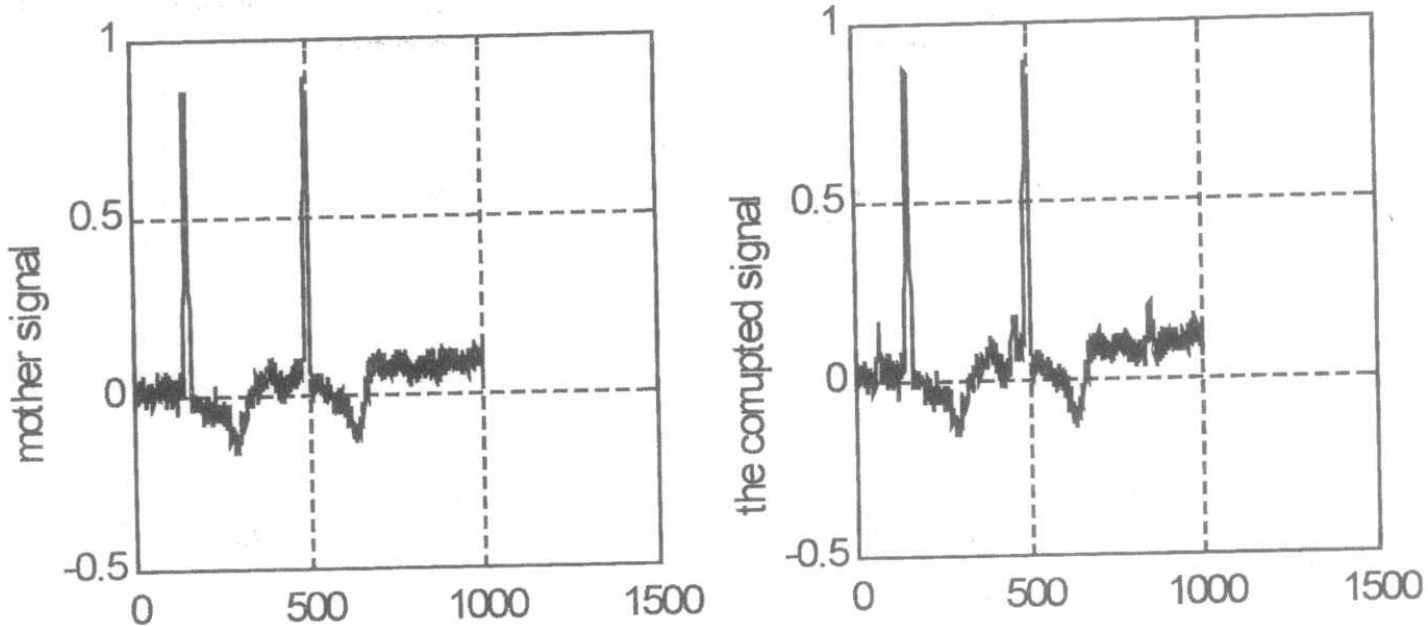

figure a the i/p mother signal time

figure $b$ the comupted fetus signal time
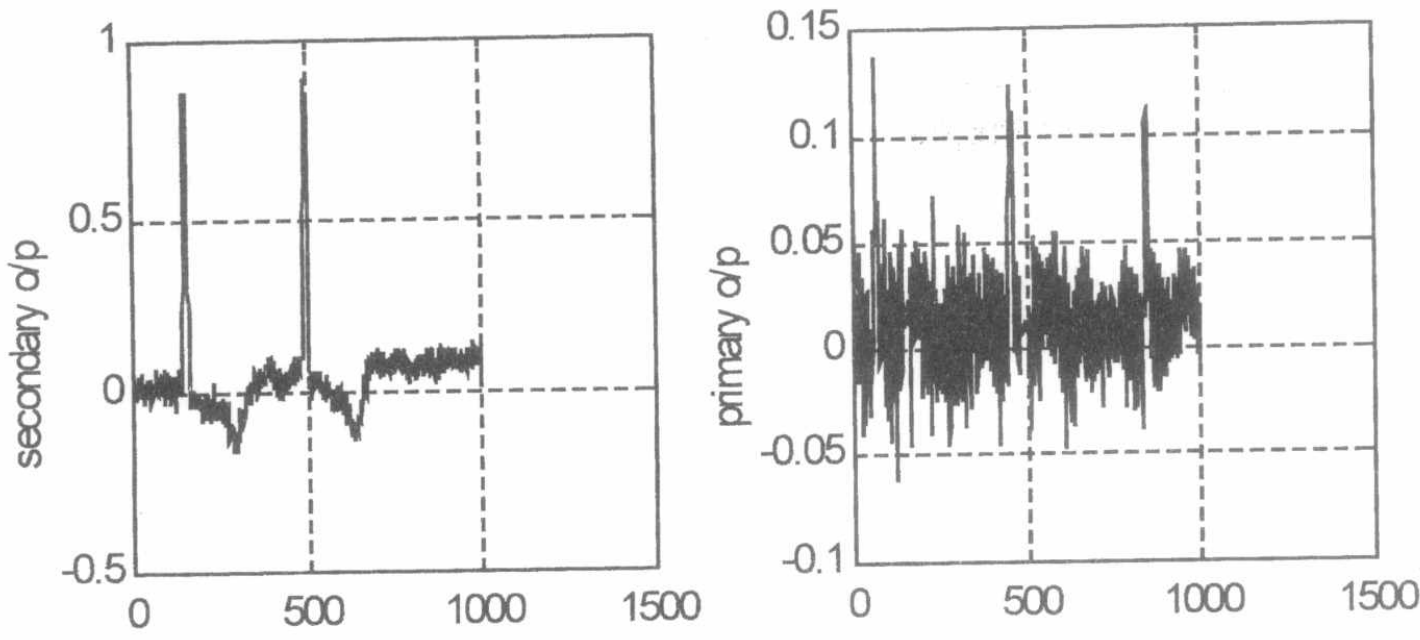

figure $\mathrm{c}$ tho $\mathrm{o} / \mathrm{p}$ mother signal time figure $\mathrm{d}$ the $\mathrm{o} / \mathrm{p}$ fetus signal

time

Figure 5 The ECG signals waveform for the fetus ECG detector.

The mother ECG signal is represented by a real ECG heart signal as depicted in figure (5-a). The composite maternal ECG signal represent the primary signal to the noise canceller as depicted in figure (5-b). The adaptive filter has tow output, the first one is a good estimate of the mother ECG signal as depicted in figure (5-c). And the second one is a good estimate of the fetus ECG signal as depicted in figure (5-d) The mother signal amplitude equal to 10 times than the fetus signal, INSR $=-16 \mathrm{~dB}, \mathrm{OSNR}=4 \mathrm{~dB}$ and the improvement factor equal $20 \mathrm{~dB}$. 
Proceedings of the $2^{\text {nd }}$ ICEENG Conference, 23-25 Nov. 1999

\begin{tabular}{|l|l|}
\hline $\mathrm{BE}-4$ & 8 \\
\hline
\end{tabular}

\section{CONCLUSIONS}

The application of the adaptive filtering in the ECG detection and enhancement has a great benefit. This is due to the fact that the usage of the classical methods fails to detect and enhance the ECG signal properly. It is clear that the proposed scheme has succeeded to detect the fetus signal that is severely corrupted with the mother signal at a very low input signal to the interference ratio Also, the proposed scheme provides a large improvement factor in the output SNR and the detected signals have no distortion.

\section{REFERENCES}

[1] Stephen Azevedo, and Richard L.Longini, " Abdominal-lead fetal electcrocardiographic R-wave enhancement for heart rate determination, " IEEE Vol. BME-27,NO.5, May 1980.

[2] Partha Pratim Kanjilal, Sarbani Palit, and Goutam Saha "Fetal ECG extraction using singular value decomposition "“ IEEE, Vol. BME-44,NO.1, January 1997. [3] Piet Bergveld, Arjan J.Kolling, and Jan H.J.Peuscher. "Real time fetal ECG recording". IEEE transaction on biomedical engineering, vol. BME 33, No.5, may 1986. [4] B. Widrow et al, "Adaptive noise canceling :Principles and applications," Proc: IEEE, Vol. 63,pp1692-1716,dec.1975 